\title{
Acromion-axillary Nerve Distance and Its Relation to the Humeral Length in the Prediction of the Axillary Nerve Position During the Anterolateral Deltoid- splitting Approach in the Treatment of Proximal Humerus Fractures: a Clinical Study
}

mehmet demirel ( $\nabla$ dr88.mehmet.demirel@gmail.com )

Istanbul University: Istanbul Universitesi

Cem Yıldırım

Başakşehir çam ve sakura City Hospital

Erhan Bayram

Gaziosmanpaşa medical park Hospital

Mehmet Ekinci

Haseki Training and Research Hospital: Istanbul Haseki Egitim Ve Arastirma Hastanesi

Murat Yilmaz

Haseki Training and Research Hospital: Istanbul Haseki Egitim Ve Arastirma Hastanesi

\section{Research Article}

Keywords: Axillary nerve, trans-deltoid approach, deltoid-splitting approach, iatrogenic nerve injury, safe zone

Posted Date: January 12th, 2022

DOI: https://doi.org/10.21203/rs.3.rs-1223482/v1

License: (c) (i) This work is licensed under a Creative Commons Attribution 4.0 International License.

Read Full License 


\section{Abstract}

\section{Background}

Because of the broad anatomical variation in the course of the axillary nerve, several cadaveric studies have investigated the acromion-axillary nerve distance and its association with the humeral length to predict the axillary nerve location. This study aimed to analyze the acromion-axillary nerve distance (AAND) and its relation to the arm length $(A L)$ in patients who underwent internal plate fixation for proximal humerus fractures.

\section{Methods}

The present prospective study involved 37 patients ( 15 female, 22 male; the mean age $=51$ years, age range $=19$ to 76 ) with displaced proximal humerus fractures who were treated by open reduction and internal fixation. After anatomic reduction and fixation was achieved, the following parameters were measured in each patient before wound closure without making an extra incision or dissection: (1) the distance from the anterolateral edge of the acromion to the course of axillary nerve was recorded as the acromion-axillary nerve distance and (2) the distance from the anterolateral edge of the acromion to the lateral epicondyle of the humerus was recorded as arm length. The ratio of AAND to AL was then calculated and recorded as the axillary nerve index.

\section{Results}

The mean AAND was $6 \pm 0.36 \mathrm{~cm}$ (range $=5.5-6.6$ ), and the mean arm length was $32.91 \pm 2.9 \mathrm{~cm}$ (range $=24-38$ ). The mean axillary nerve ratio was $0.18 \pm 0.02$ (range $=0.16$ to 0.23 ). There was a significant moderate positive correlation between $\operatorname{AL}$ and AAND $(p=0.006 ; r=0.447)$. The axillary nerve location was predictable in only $18 \%$ of the patients.

\section{Conclusion}

During the anterolateral deltoid-splitting approach to the shoulder joint, $5.5 \mathrm{~cm}$ from the anterolateral edge of the acromion could be considered as a safe zone for the prevention of possible axillary nerve injury.

\section{Background}

In the operative treatment of proximal humerus fractures, the deltopectoral approach is still the most widely used approach for internal plate fixation. However, this traditional approach offers limited access to the posterolateral aspect of the proximal humerus that may render reduction of a retracted greater tuberosity fragment and plate placement difficult [1] Alternatively, the anterolateral deltoid-splitting approach can provide direct access and excellent visualization of the greater tuberosity and the plating area, with minimal soft-tissue dissection [2, 3], but there is an increased risk for axillary nerve injury, accounting for 6 to $10 \%$ of all iatrogenic nerve injuries to the brachial plexus [4-8]. 
Although it is generally accepted that the axillary nerve crosses the humerus horizontally nearly $50 \mathrm{~mm}$ distal to the acromion in clinical practice, various anatomical studies have defined a broad range of safe zones for deltoid-splitting approaches, varying from 30 to $70 \mathrm{~mm}$ distally to the acromion [6, 9-11]. Furthermore, it has been shown that a safe zone for a nerve may change in size as per the extremity length [12]. Because of the large anatomical variation in the course of the axillary nerve from one individual to another, several cadaveric studies have explored the acromion-axillary nerve distance and its association with the humeral length to predict the axillary nerve location [13-16]. Nonetheless, to the best of our knowledge, the relationship between the axillary nerve location and humeral length has not been investigated in a clinical setting to date.

This study aimed to analyze the acromion-axillary nerve distance (AAND) and its relation to the arm length (AL) in patients who underwent internal plate fixation for proximal humerus fractures. The authors hypothesized that acromion-axillary nerve distance has a significant correlation with the humeral length and can be used to predict the axillary nerve location during anterolateral deltoid-splitting approach.

\section{Methods}

The present prospective study involved 37 patients ( 15 female, 22 male; the mean age $=51$ years, age range $=19$ to 76 ) with displaced proximal humerus fractures who were treated by open reduction and internal fixation at a single tertiary trauma referral center from January 2017 to May 2019. According to the Neer classification system (17), there were 15 two-part (41\%), 20 three-part (54\%), and two four-part $(5 \%)$ humerus fractures. Inclusion criteria were patients aged $>18$ years, with proximal humerus fractures without a previous history of shoulder surgery. Exclusion criteria were patients with polytrauma, pathological fracture, concomitant fracture of the same upper extremity, limb discrepancy, or congenital deformity. An informed consent was obtained from all the patients preoperatively; ethical approval was obtained from the institutional ethical committee (88-2021, 06.10.2021).

\section{Operative technique}

All surgical procedures were performed by a single experienced orthopedic trauma surgeon within a week of the injury, using the anterolateral deltoid-splitting approach. All the operations were performed under general anesthesia. The patients were placed in a beach-chair position, and bony landmarks were marked before making the incision. A longitudinal incision was made from the anterolateral edge of the acromion, which extended distally along the long axis of the humerus, and dissection was performed between the anterior and middle thirds of the deltoid muscle fibers. The axillary nerve was then palpated and visualized carefully. After ensuring adequate protection of the axillary nerve, the dissection was extended distally. The exposed region of the shoulder was divided into two parts by the axillary nerve. While the superior part was used for reduction of the fracture, the distal part was used for fixing the plate to the humeral shaft. Later, the fracture was reduced, and Kirschner wires were inserted for temporary fixation. The anatomic proximal humerus plate was then placed under the axillary nerve, and the rotator cuff was repaired if required. The final position was checked using fluoroscopy. The wound was closed in 
layers, and a drain inserted inside the subcutaneous tissue. Postoperatively, the arm was placed in a sling for controlled physical therapy.

\section{Outcome measures}

Within the routine steps of the planned operation, after anatomic reduction and fixation was achieved, the following parameters were measured in each patient before wound closure without making an extra incision or dissection: (1) the distance from the anterolateral edge of the acromion to the course of axillary nerve was recorded as the acromion-axillary nerve distance (Fig. 1), and (2) the distance from the anterolateral edge of the acromion to the lateral epicondyle of the humerus was recorded as AL [13]. The ratio of AAND to AL was then calculated and recorded as the axillary nerve index for each patient as described by Çetik et al.[13]. The correlation between AAND and AL was also investigated.

\section{Statistical Analysis}

All statistical analyses were performed using SPSS 25.0 software (SPSS Inc., Chicago, IL, USA). A p value of 0.05 was considered as statistically significant. Descriptive statistics were given as mean, standard deviation, percent, lowest $(\mathrm{min})$ and highest $(\mathrm{max})$ values. The Kolmogorov-Smirnov test was used to verify the normal distribution of the variables. The correlation between AAND and AL was evaluated using the Spearman correlation analysis in a linear regression model. The level of correlation was interpreted according to Guilford's interpretation [17].

\section{Results}

The mean AAND was $6 \pm 0.36 \mathrm{~cm}$ (range $=5.5$ to $6.6 \mathrm{~cm}$ ), and the mean AL was $32.9 \pm 2.9 \mathrm{~cm}$ (range $=$ 24 to $38 \mathrm{~cm}$ ). The mean axillary nerve index was $0.18 \pm 0.02$ (range $=0.16$ to 0.23 ) (Table 1). A significant moderate positive correlation was identified between AL and ANND $(p=0.006 ; r=0.447)$ (Fig. 2). We were able to predict the location of the axillary nerve in $18 \%$ of the patients using the regression analysis.

Table 1

Demographic data of the study participants

\begin{tabular}{|ll|}
\hline Number of the patients & 37 \\
\hline Age (years), mean & 51 (range $=17-76)$ \\
\hline Gender (Male/Female) & $22 / 15$ \\
\hline AAND $(\mathrm{cm})$, mean \pm SD & $6 \pm 0.36 \mathrm{~cm}$ (range = 5.5-6.6) \\
\hline AL $(\mathrm{cm})$, mean \pm SD & $32.91 \pm 2.9 \mathrm{~cm}($ range $=24-38)$ \\
\hline Axillary nerve index $(A A N D / A L)$, mean $\pm S D$ & $0.18 \pm 0.02(0.16-0.23)$ \\
\hline AAND = Acromion-axillary nerve distance; AL = Arm Length; SD = Standard Deviation \\
\hline
\end{tabular}




\section{Discussion}

Although the anterolateral deltoid-splitting approach can ensure direct access and excellent visualization of the plating area in the management of proximal humerus fractures [2,3], there is an increased risk for axillary nerve injury, which is the most common neurological complication associated with surgery of proximal humerus fractures $[6,18,19]$. Accordingly, defining the safe zone for the axillary nerve is important to avoid iatrogenic injury. However, various anatomical studies have defined a broad range of safe zones for deltoid-splitting approaches, varying from 30 to $70 \mathrm{~mm}$ distally to the acromion [6, 9-11]. Because of the broad anatomical variation in the course of the axillary nerve, the acromion-axillary nerve distance and its association with the humeral length were investigated to predict the axillary nerve location [13-16] in some cadaveric studies. Nonetheless, according to our review of the literature, the relationship between the axillary nerve location and humeral length has not been investigated in a clinical setting to date.

The present study aimed to describe a safe area for executing the anterolateral deltoid split approach during open reduction-plate fixation for managing patients with proximal humerus fractures. We found that ANND was $6.0 \pm 0.36 \mathrm{~cm}$, which was moderately correlated with AL. However, ANND could be predicted according to AL in only $18 \%$ of the patients.

Numerous studies have attempted to measure ANND and found significant variations with a range of 4.5 to $7.5 \mathrm{~cm}[10,13-15,20,21]$. Kongcharoensombat et al. [14] calculated the mean distance of the axillary nerve from the anterolateral acromion as $6.39 \mathrm{~cm}$ (ranging from 4.6 to $8.2 \mathrm{~cm}$ ), and Cetik et al. [13] found the distance of the axillary nerve from the anterolateral acromion to be $6.08 \mathrm{~cm}$ (ranging from 5.20 to $6.90 \mathrm{~cm}$ ). Both previous studies observed significant correlation between the distance of the axillary nerve from the anterolateral acromion and humeral length. In contrast to the cadaveric studies of Kongcharoensombat [14] and Cetik et al. [13], the present study was conducted in a clinical setting, and all measurements were performed intraoperatively after the anatomic reduction and fixation were completed. In this regard, our study is advantageous over the existing previous cadaveric studies in the literature.

While using the anterolateral approach for proximal humeral fractures, the plate should be inserted under the axillary nerve so that the nerve could be dissected carefully, and potential injury could be prevented. Also, the shortest distance should be taken into consideration during dissection to minimize the risk of probable axillary nerve injury. We measured the minimum distance of the axillary nerve to be $5.5 \mathrm{~cm}$ from the acromion. Hence, this distance could be considered as a safe zone according to the findings of the present study. In the study by Cetik et al. [13], this distance was measured as $5.2 \mathrm{~cm}$. However, this data contradicts the findings of Kongcharoensombat et al. [14] because the axillary nerve was found located at $<5 \mathrm{~cm}$ in $13 \%$ of the cadaver shoulders.

In our study, the calculated mean axillary nerve index was lower than that given by Cetik et al. [13] and Kongcharoensombat et al. [21]. The exact prediction ratio of the location of the axillary nerve according 
to the humeral length of the patients was $18 \%$, which was less than the expected value. Therefore, we believe that it would be safer to use the distance instead of the ratio.

Our study has several limitations. First, the number of patients who participated in the study was less. Second, the measurements were made using a manual caliper, thereby giving room for human errors. Third, all the measurements were performed after the anatomic reduction was completed. However, in case of deformity due to proximal humerus fracture before reduction was performed during the exposure, this distance is likely to be shortened.

\section{Conclusions}

Evidence from this study has demonstrated that during the anterolateral deltoid-splitting approach to the shoulder joint, $5.5 \mathrm{~cm}$ from the anterolateral edge of the acromion could be considered as a safe zone for the prevention of possible axillary nerve injury. Predicting the location of the axillary nerve using the $A L$ was possible in only $18 \%$ of the patients; thus, it would be safer to use the distance of $5.5 \mathrm{~cm}$ instead of relying on the axillary nerve index.

\section{Abbreviations}

AAND: Acromion-axillary nerve distance

AL: Arm length

\section{Declarations}

\section{Ethical approval and consent to participate}

This study was approved by the institutional review board of our institution and was carried out in accordance with the Declaration of Helsinki. Informed consent was obtained from all individual participants included in the study.

\section{Consent for publication}

Patients signed informed consent regarding publishing their data and photographs.

\section{Competing interests}

All authors promise that there is no competing interest to disclose.

\section{Funding}


Not applicable.

\section{Authors' contributions}

CY: Conceptualization; Data curation; Methodology; Investigation

MD: Validation; Writing - original draft

EB: Methodology; Formal analysis; Writing - original draft,

ME: Formal analysis; Supervision; Validation; Writing - review \& editing

MY: Supervision; Validation

\section{Availability of data and materials}

The data used and/or analysed during the current study are available from the corresponding author or the first author on reasonable request.

\section{References}

1. Xie L, Zhang Y, Chen C, Zheng W, Chen H, Cai L. Deltoid-split approach versus deltopectoral approach for proximal humerus fractures: A systematic review and meta-analysis. Orthop Traumatol Surg Res. 2019;105(2):307-16.

2. Traver JL, Guzman MA, Cannada LK, Kaar SG. Is the axillary nerve at risk during a deltoid-splitting approach for proximal humerus fractures? J Orthop Trauma. 2016;30(5):240-4.

3. Zhang J, Moore AE, Stringer MD. latrogenic upper limb nerve injuries: a systematic review. ANZ J Surg. 2011;81(4):227-36.

4. Eakin CL, Dvirnak P, Miller CM, Hawkins RJ. The relationship of the axillary nerve to arthroscopically placed capsulolabral sutures. Am J Sports Med. 1998;26(4):505-9.

5. Lynch NM, Cofield RH, Silbert PL, Hermann RC. Neurologic complications after total shoulder arthroplasty. J Shoulder Elbow Surg. 1996;5(1):53-61.

6. Perlmutter GS. Axillary nerve injury. Clin Orthop Relat Res. 1999(368):28-36.

7. Smith J, Berry G, Laflamme Y, Blain-Pare E, Reindl R, Harvey E. Percutaneous insertion of a proximal humeral locking plate: an anatomic study. Injury. 2007;38(2):206-11.

8. Tubbs RS, Tyler-Kabara EC, Aikens AC, Martin JP, Weed LL, Salter EG, Oakes WJ. Surgical anatomy of the axillary nerve within the quadrangular space. J Neurosurg. 2005;102(5):912-4.

9. Bryan WJ, Schauder K, Tullos HS. The axillary nerve and its relationship to common sports medicine shoulder procedures. Am J Sports Med. 1986;14(2):113-6. 
10. Burkhead W Jr, Scheinberg R, Box G. Surgical anatomy of the axillary nerve. J Shoulder Elbow Surg. 1992;1(1):31-6.

11. Duparc F, Bocquet G, Simonet J, Freger P. Anatomical basis of the variable aspects of injuries of the axillary nerve (excluding the terminal branches in the deltoid muscle). Surg Radiol Anat. 1997;19(3):127-32.

12. Eksioglu F, Uslu M, Gudemez E, Atik OS, Tekdemir I. Reliability of the safe area for the superior gluteal nerve. Clin Orthop Relat Res. 2003;412:111-6.

13. Cetik O, Uslu M, Acar HI, Comert A, Tekdemir I, Cift H. Is there a safe area for the axillary nerve in the deltoid muscle?: a cadaveric study. J Bone Joint Surg Am. 2006;88(11):2395-9.

14. Kongcharoensombat W, Wattananon P. Risk of axillary nerve injury in standard anterolateral approach of shoulder: cadaveric study. Malays Orthop J. 2018;12(3):1.

15. Rotari V, Moussallem CD, David E, Mertl P, Havet E. Position of the anterior branch of the axillary nerve in relation to the humeral bone length. Am J Orthop. 2012;41(10):452-4.

16. Sung C-M, Roh GS, Sohn H-J, Park HB. Prediction of the location of the anterior branch of the axillary nerve, using correlations with physical factors: a cadaveric study. J Shoulder Elbow Surg. 2013;22(11):e9-16.

17. Tredoux C, Durrheim K: Number, hypotheses \& conclusions: A course in statistics for the social sciences. Cape Town. University of Cape Town Press Triguero, A, Córcoles, D, \& Cuerva, MC (2014) Persistence of innovation and firm's growth: evidence from a panel of SME and large Spanish manufacturing firms Small Business Economics 2002, 43(4):787-804.

18. Shaw A, Milne A, Christie J, Jenkins AM, Murie J, Ruckley C. Vascular trauma of the upper limb and associated nerve injuries. Injury. 1995;26(8):515-8.

19. Visser CP, Coene LNJ, Brand R, Tavy DL. Nerve lesions in proximal humeral fractures. J Shoulder Elbow Surg. 2001;10(5):421-7.

20. Kamineni S, Ankem H, Sanghavi S. Anatomical considerations for percutaneous proximal humeral fracture fixation. Injury. 2004;35(11):1133-6.

21. Kontakis GM, Steriopoulos K, Damilakis J, Michalodimitrakis E. The position of the axillary nerve in the deltoid muscle: A cadaveric study. Acta Orthop Scand. 1999;70(1):9-11.

\section{Figures}




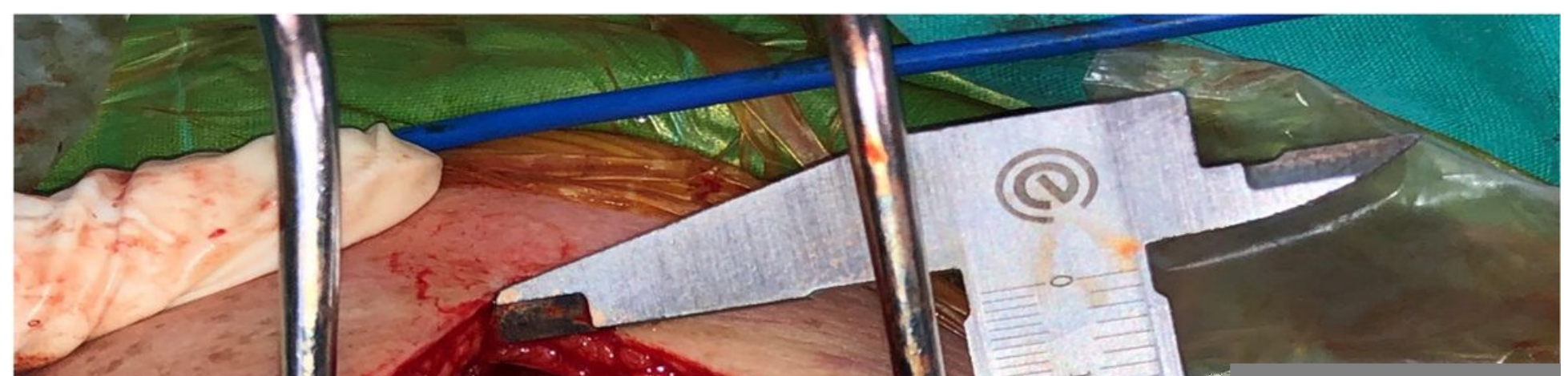

\section{Figure 1}

Representative figure showing the intraoperative measurement method of the distance between the anterolateral edge of the acromion and the axillary nerve (blue arrow) using a caliper. 


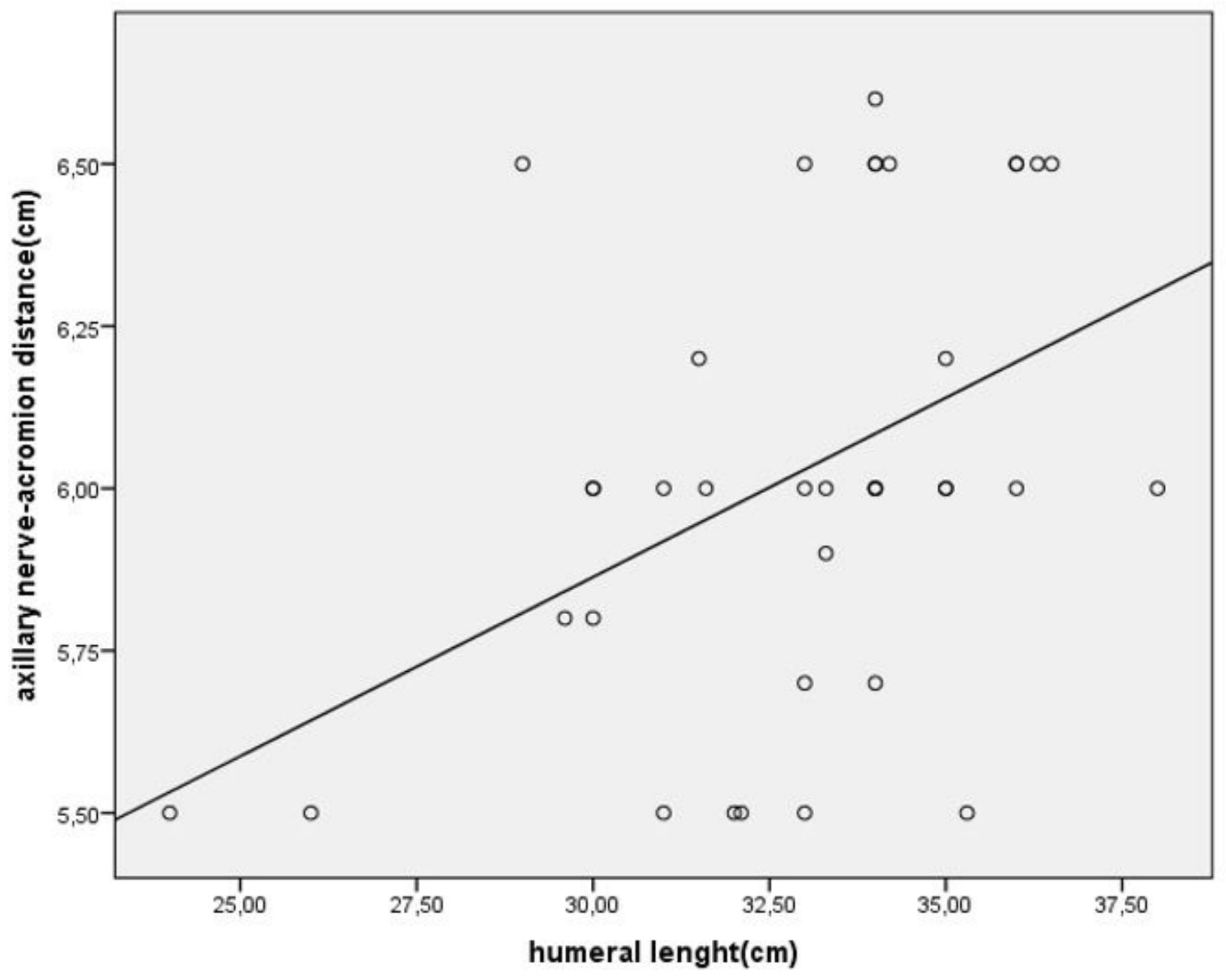

Figure 2

Graph illustrating the correlation between arm length and axillary nerve distance from the anterolateral edge of the acromion. 